\title{
Defining the spicity
}

\author{
by Rui Xin Huang ${ }^{1,2}$
}

\begin{abstract}
Spicity is defined as a thermodynamic variable whose isopleths are "perpendicular" to those of density in the $(T, S)$ diagram. A new set of Matlab codes are defined based on the following criteria: First, the isopleths of spicity should be orthogonal to those of density. Second, the ocean is vertically separated into many pressure levels, and the zero point of spicity is set for the water mass with the mean salinity and temperature at each pressure level. This new definition of spicity can provide more accurate information of the water mass properties for the study of thermohaline perturbations.
\end{abstract}

\section{Introduction}

In the common practice of physical oceanography, seawater is treated as a two-component thermodynamic system, and its thermodynamic state can be uniquely determined in terms of three independent variables. The most frequently used independent variables are temperature, salinity and pressure. In the study of physical oceanography, oceanic circulation is often examined in terms of seawater property distribution on some thermodynamic surfaces (such as a pressure surface, a potential density surface, or a neutral surface); thus, the thermodynamics of seawater can be described in terms of two other independent variables.

One of the most important and commonly used thermodynamic variables is the density of seawater. Density distribution in the vertical direction controls the stability of the water column, and its horizontal distribution is directly linked to the horizontal pressure gradient and thus the dynamics of ocean circulation. In addition to density, we need another thermodynamic variable to provide the additional information regarding the thermodynamic state of the system.

Ideally, the other thermodynamic quantity should be able to provide the rest of the thermodynamic information with the least amount of information overlapping with density; thus, the most desirable thermodynamic variable complementary to the density should be "orthogonal" or "perpendicular" to the constant density curves in the $(T, S)$ diagram, as discussed in many previous studies such as Stommel (1962), Veronis (1972), and Munk (1981).

1. State Key Laboratory of Tropical Oceanography, South China Sea Institute of Oceanology, Chinese Academy of Science, Guangzhou, China.

2. Department of Physical Oceanography, Woods Hole Oceanographic Institution, Woods Hole, Massachusetts, 02543, U.S.A.email: rhuang@whoi.edu 
In particular, Veronis argued that a sensible choice of such a thermodynamic variable is that its contours should be perpendicular to density contours; he also presented some technical details of defining such a variable. Munk (1981) introduced the term spiciness which has been widely used since. We will use a slightly different name spicity for this thermodynamic variable. Since density and salinity have been widely used as the thermodynamic variables, the term spicity seems a better replacement for the spiciness recommended by Munk.

The most desirable definition of spicity should meet the following criteria. First, its isopleths should be perpendicular to the constant density contours. Second, spicity is defined up to a constant; such a constant should be so chosen that the sign (positive or negative) should indicate the fundamental character of water mass, i.e. whether it is warm/salty or cold/fresh. Up till now, the only publically available subroutine for spiciness was proposed by Flament (2002). However, his definition does not meet the criteria listed above; therefore, we will discuss a new definition which can better meet these criteria.

Thermohaline circulation in the ocean is directly linked to the transportations and diffusion of heat and salt. Thermohaline perturbations can appear in forms over a wide range of spatial and temporal scales. One of the well-known phenomena is double diffusion, including salt finger and diffusive layering. Such phenomena have been discussed in many previous studies, e.g. Stern and Turner (1969) and Schmitt (1994); a collection of papers written by experts in this field was published in Progress of Oceanography in 2003. Spicity is a thermodynamic variable which can be used as a tool in the study of double diffusion and other thermohaline processes.

This short contribution is organized as follows. In Section 2, we re-examine the definition of spicity and introduce a new definition of spicity which better meets the basic criteria. The newly defined spicity is applied to the World Oceans in Section 3. The advantages of using the newly defined spicity are demonstrated by comparing the section maps obtained from the previously defined spiciness and the newly defined spicity. Finally, concluding remarks are presented in Section 4.

\section{Defining the spicity}

\section{a. Previous definitions}

Veronis (1972) discussed how to define such a thermodynamic variable, which he denoted as $\tau$, but gave no name. Jackett and McDougal (1985) postulated a definition of spiciness, in which the orthogonal property is required for a single spiciness isopleths. No code related to these studies is available for public use. Although most previous discussions were focused on globally defined spiciness, locally defined spiciness can be used as well. For example, Rudnick and Martin (2002) discussed density ratio in the upper layer of the World Oceans. In fact, since their discussion was focused on small-scale phenomena spicity can be defined by a simple and straightforward integration of the orthogonal condition discussed below. For application to the World Oceans, Flament (2002) postulated a slightly different definition of spiciness. Instead of defining a thermodynamic variable whose contours are perpendicular 
to the density contours, his definition of spiciness requires that, at any given point in the $(\Theta, S)$ space, the slope of the isopycnals and spiciness isopleths are equal and of opposite signs. A Matlab code is available, in which the spiciness is defined as a polynomial

$$
\pi(\Theta, S)=\sum_{i=0}^{5} \sum_{j=0}^{5} b_{i j} \Theta^{i}(S-35)^{j} .
$$

The spiciness defined in this way requires two more parameters. The first is the reference pressure level which is set to $p=0$ as the pivotal choice. The second one is an arbitrary constant for the spiciness. Flament chose a point in the $(\Theta, S)$ diagram with $\Theta=0^{\circ} \mathrm{C}$ and $S=35$ as the zero point for spiciness. This choice of the reference value for spiciness is inconvenient because a water mass with temperature slightly higher than zero degree is assigned a positive value of spiciness. However, such a water on the sea surface is not warm at all; thus, such a definition is less than desirable.

\section{A new definition}

First of all, in order to define the so-called perpendicular or orthogonal of two families of curve, we must use the same dimension for both axes of the $(T, S)$ diagram. In such a coordinates, we can compare gradients at different directions in the $(T, S)$ space. In this study we will use the following pair of variable

$$
\begin{aligned}
& x=\rho_{0} \beta_{0} S, \frac{\partial}{\partial x}=\frac{1}{\rho_{0} \beta_{0}} \frac{\partial}{\partial S} ; \\
& y=\rho_{0} \alpha_{0} T, \frac{\partial}{\partial y}=\frac{1}{\rho_{0} \alpha_{0}} \frac{\partial}{\partial T} .
\end{aligned}
$$

where $\rho_{0}$ is the mean reference density, $\alpha_{0}$ and $\beta_{0}$ are the thermal expansion coefficient and saline contraction coefficient, both averaged over the domain where the spicity is defined. This definition guarantees that the $\mathrm{x}$-axis and $\mathrm{y}$-axis have the same dimension. In this coordinates the density gradient is equal to

$$
\nabla \rho=\vec{i} \frac{\partial \rho}{\partial x}+\vec{j} \frac{\partial \rho}{\partial y}=\vec{i} \frac{1}{\rho_{0} \beta_{0}} \frac{\partial \rho}{\partial S}+\vec{j} \frac{1}{\rho_{0} \alpha_{0}} \frac{\partial \rho}{\partial T}=\vec{i} \frac{\beta}{\beta_{0}}-\vec{j} \frac{\alpha}{\alpha_{0}} .
$$

On the sea surface, the net pressure is zero, i.e. $p=0$, thus the thermodynamic state of seawater can be expressed in terms of two variables $(T, S)$. In the traditional way, density is used as a dynamical variable; the best choice of the other thermodynamic variable is the one which varies along the density isopleths. This variable is called spicity, denoted as $\pi$. By definition, the gradient vectors of density and spicity should be perpendicular

$$
\nabla \rho \cdot \nabla \pi=0 .
$$


Accordingly, we use constraint (4) to introduce the spicity. Specifically, we require that the gradient of spicity should satisfy

$$
\nabla \pi=-\vec{i} \frac{\partial \rho}{\partial y}+\vec{j} \frac{\partial \rho}{\partial x}=-\vec{i} \frac{1}{\rho_{0} \alpha_{0}} \frac{\partial \rho}{\partial T}+\vec{j} \frac{1}{\rho_{0} \beta_{0}} \frac{\partial \rho}{\partial S} .
$$

It is readily seen that the vector field associated with the gradient of spicity is nondivergent, i.e.

$$
\nabla \cdot(\nabla \pi)=0
$$

The next step is to construct the spicity field from its gradient, Eq. (5), i.e.

$$
\begin{aligned}
& \pi_{x}=-\partial \rho / \partial y=-\frac{1}{\rho_{0} \alpha_{0}} \frac{\partial \rho}{\partial T}=\frac{\alpha}{\alpha_{0}}>0, \\
& \pi_{y}=\partial \rho / \partial x=\frac{1}{\rho_{0} \beta_{0}} \frac{\partial \rho}{\partial S}=\frac{\beta}{\beta_{0}}>0
\end{aligned}
$$

By definition, spicity has the same dimension as density, so that it can be written in the same unit as density, i.e. $\mathrm{kg} / \mathrm{m}^{3}$.

The construction of spicity can be started from a chosen fixed point $\left(x_{0}, y_{0}\right)$ in the $(T, S)$ space, and Eq. (7) can be used to define spicity through integration. Both the thermal expansion coefficient $\alpha$ and the saline contraction coefficient $\beta$ vary with ambient conditions, so that it is difficult to obtain a set of curves that are strictly perpendicular to density isopleths. To obtain spicity curves that closely approximate orthogonality to the density isopleths, we begin the y-integration from the reference point $x=x_{0}, y=y_{0}$ (which corresponds to the mean temperature and salinity at a given reference pressure level) upward and downward.

$$
\pi\left(x_{0}, y\right)=\pi\left(x_{0}, y_{0}\right)+\int_{y_{0}}^{y} \pi_{y}\left(x_{0}, y^{\prime}\right) d y^{\prime}=\pi\left(x_{0}, y_{0}\right)+\rho_{0} \alpha_{0} \int_{y_{0}}^{y} \frac{\beta\left(x_{0}, y\right)}{\beta_{0}} d T .
$$

Secondly, we integrate Eq. (7) in the $\mathrm{x}$-direction from the now given value of spicity along the $x=x_{0}$ line

$$
\pi(x, y)=\pi\left(x_{0}, y\right)+\int_{x_{0}}^{x} \pi_{x}\left(x^{\prime}, y\right) d x^{\prime}=\pi\left(x_{0}, y\right)+\rho_{0} \beta_{0} \int_{x_{0}}^{x} \frac{\alpha\left(x^{\prime}, y\right)}{\alpha_{0}} d S
$$

Spicity defined in this way leaves an arbitrary constant $\pi\left(x_{0}, y_{0}\right)$ at the reference point. A sensible choice is to set the spicity corresponding to the mean salinity and temperature at a given reference pressure level as zero. In this way, a water parcel with positive (negative) spicity indicates that this water parcel is warm and salty (cold and fresh) in comparison to the reference water parcel, and there is no ambiguity about the meaning of the spicity. Although horizontal mean salinity does not vary much with depth, temperature averaged on different levels varies greatly with depth. For example, a water parcel of $5^{\circ} \mathrm{C}$ on the sea surface is quite cold, but the same water parcel in the deep ocean is considered very warm. 
Therefore, in order to make the spicity a good indicator for the thermohaline circulation in the World Oceans, we use the horizontal mean temperature and salinity as the reference temperature and salinity at each depth.

The newly available climatology WOA09 data (Antonov et al., 2010; Locarnini et al., 2010) is a gridded data based on the traditional z-coordinate. However, the applications require information of pressure for each grid; thus, this data have been converted into a dataset based on the pressure coordinate. The corresponding area-mean reference temperature and salinity are set to the closest value of the temperature and salinity grid in our calculation as listed in Table 1.

At all depths spicity is defined for the temperature range of $\left[-2^{\circ} \mathrm{C}, 30^{\circ} \mathrm{C}\right]$, and salinity range is $[33,37]$. The $(T, S)$ space has a $641 \times 401$ grid points. As discussed above, the spicity is calculated accordingly, with a zero value set at the mean temperature and salinity for each reference pressure level. The spicity values over this $641 \times 401$ grid are then least-square fit to the following six-order polynomial

$$
\begin{aligned}
\pi(S, \Theta)= & a_{1}+a_{2} S+a_{3} \Theta+a_{4} S^{2}+a_{5} S \Theta+a_{6} \Theta^{2} \\
& +a_{7} S^{3}+a_{8} S^{2} \Theta+a_{9} S \Theta^{2}+a_{10} \Theta^{3} \\
& +a_{11} S^{4}+a_{12} S^{3} \Theta+a_{13} S^{2} \Theta^{2}+a_{14} S \Theta^{3}+a_{15} \Theta^{4} \\
& +a_{16} S^{5}+a_{17} S^{4} \Theta+a_{18} S^{3} \Theta^{2}+a_{19} S^{2} \Theta^{3}+a_{20} S \Theta^{4}+a_{21} \Theta^{5} \\
& +a_{22} S^{6}+a_{23} S^{5} \Theta+a_{24} S^{4} \Theta^{2}+a_{25} S^{3} \Theta^{3}+a_{26} S^{2} \Theta^{4}+a_{27} S \Theta^{5}+a_{28} \Theta^{6} .
\end{aligned}
$$

Since double precision was used in the calculation, the root-mean square error of this polynomial fitting is in the range of $(3-10) \times 10^{-6}\left(\mathrm{~kg} / \mathrm{m}^{3}\right)$; thus, the resulting polynomial has a high accuracy for practical applications.

These polynomials are converted into Matlab programs named as sw_spicity_N.m, where $\mathrm{N}$ stands for the following values: $0,0.1,0.2,0.3,0.4,0.5,0.6,0.7,0.8,0.9,1,1.1,1.2,1.3$, $1.4,1.5,1.75,2,2.5,3,3.5,4,4.5,5,5.5$, Table 1 . Thus, spicity is defined as a function of $(S, T, p)$, where $S$ is salinity, $T$ is in-situ temperature and $p$ is the in-situ pressure. The Matlab functions defined above first convert the in-situ temperature to a potential temperature using the corresponding in-situ pressure and reference pressure; then the corresponding spicity is calculated. Since each Matlab program is based on the algorithms discussed above, where the potential temperature defined by using the corresponding reference pressure, such spicity can be called as potential spicity with the corresponding reference pressure.

The fourth column in Table 1 is the mean value of $\beta_{0} / \alpha_{0}$, which indicates the mean slope of density-isolines on the $(\Theta, S)$ diagram at each pressure level. As shown in Table 1, the mean slope of density-isolines declines with increasing pressure, i.e., density-isolines rotate clockwise with increasing pressure. This rotation can be seen clearly in Figure 1; however, one should also notice that in order to plot these panels with the same y-axis height, the corresponding length of the $\mathrm{x}$-axis is gradually reduced with increasing pressure level. 
Table 1. Zero point for the potential spicity defined at different reference pressure levels, where $\beta_{0} / \alpha_{0}$ indicates the ratio of the mean haline contraction coefficient and the mean thermal expansion coefficient at each pressure level, $\sigma_{t, 0}$ indicates the in-situ density for a water mass with the corresponding mean salinity $S_{0}$ and temperature $T_{0}$ at each pressure level, all based on WOA09.

\section{Reference}

Potential pressure

\begin{tabular}{|c|c|c|c|c|c|c|}
\hline spicity & (1000db) & $S_{0}$ & $T_{0}\left({ }^{\circ}\right) \mathrm{C}$ & $\beta_{0} / \alpha_{0}$ & $\sigma_{t, 0}\left(\mathrm{~kg} / \mathrm{m}^{3}\right)$ & $\left(10^{-6} \mathrm{~kg} / \mathrm{m}^{3}\right)$ \\
\hline & 0 & 34.72 & 3.3853 & 3.8351 & 27.6239 & 6.974 \\
\hline$\pi_{0}$ & 0 & 34.59 & 18.15 & 3.8351 & 24.9221 & 5.887 \\
\hline$\pi_{0.1}$ & 0.1 & 34.97 & 14.99 & 3.7975 & 26.6096 & 5.878 \\
\hline$\pi_{0.2}$ & 0.2 & 34.95 & 11.84 & 3.7606 & 27.4757 & 5.702 \\
\hline$\pi_{0.3}$ & 0.3 & 34.83 & 9.92 & 3.7243 & 28.1908 & 5.213 \\
\hline$\pi_{0.4}$ & 0.4 & 34.73 & 8.52 & 3.6888 & 28.8010 & 5.019 \\
\hline$\pi_{0.5}$ & 0.5 & 34.66 & 7.40 & 3.6538 & 29.3652 & 4.621 \\
\hline$\pi_{0.6}$ & 0.6 & 34.61 & 6.50 & 3.6195 & 29.9146 & 4.530 \\
\hline$\pi_{0.7}$ & 0.7 & 34.58 & 5.80 & 3.5858 & 30.4539 & 4.467 \\
\hline$\pi_{0.8}$ & 0.8 & 34.57 & 5.22 & 3.5527 & 30.9921 & 3.766 \\
\hline$\pi_{0.9}$ & 0.9 & 34.59 & 4.70 & 3.5202 & 31.5228 & 3.758 \\
\hline$\pi_{1}$ & 1.0 & 34.60 & 4.28 & 3.4883 & 32.0391 & 3.730 \\
\hline$\pi_{1.1}$ & 1.1 & 34.61 & 3.93 & 3.4569 & 32.5480 & 3.507 \\
\hline$\pi_{1.2}$ & 1.2 & 34.63 & 3.65 & 3.4261 & 33.0577 & 3.447 \\
\hline$\pi_{1.3}$ & 1.3 & 34.65 & 3.41 & 3.3958 & 33.5605 & 3.367 \\
\hline$\pi_{1.4}$ & 1.4 & 34.67 & 3.20 & 3.3661 & 34.0563 & 3.207 \\
\hline$\pi_{1.5}$ & 1.5 & 34.69 & 3.01 & 3.3368 & 34.5518 & 3.271 \\
\hline$\pi_{1.75}$ & 1.75 & 34.71 & 2.63 & 3.2658 & 35.7451 & 3.462 \\
\hline$\pi_{2}$ & 2.0 & 34.73 & 2.36 & 3.1978 & 36.9287 & 3.880 \\
\hline$\pi_{2.5}$ & 2.5 & 34.75 & 1.99 & 3.0700 & 39.2346 & 4.368 \\
\hline$\pi_{3}$ & 3.0 & 34.75 & 1.74 & 2.9523 & 41.4930 & 5.989 \\
\hline$\pi_{3.5}$ & 3.5 & 34.74 & 1.55 & 2.8437 & 43.7172 & 6.417 \\
\hline$\pi_{4}$ & 4.0 & 34.73 & 1.41 & 2.7434 & 45.9139 & 8.172 \\
\hline$\pi_{4.5}$ & 4.5 & 34.73 & 1.31 & 2.6506 & 48.0894 & 9.530 \\
\hline$\pi_{5}$ & 5.0 & 34.73 & 1.32 & 2.5646 & 50.2248 & 9.422 \\
\hline$\pi_{5.5}$ & 5.5 & 34.72 & 1.41 & 2.4849 & 52.3173 & 10.704 \\
\hline
\end{tabular}



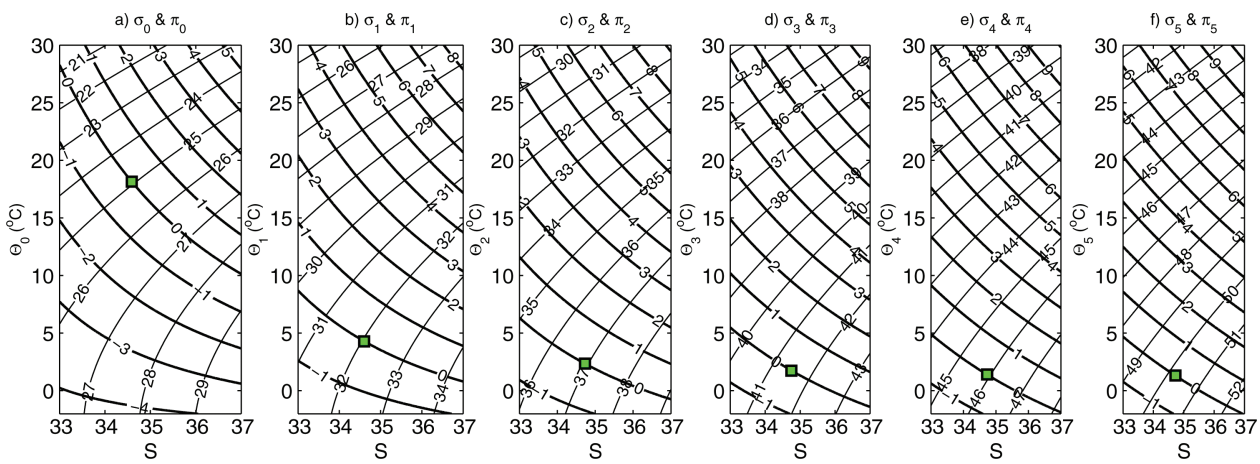

Figure 1. $\Theta-S$ diagrams, including both potential density and potential spicity, based on different reference pressures, where the potential temperature for each panel is defined by using the nominal pressure at each level as the reference pressure. The small squares indicate the mean potential temperature and salinity for each pressure level. Change in the aspect ratio of these figures reflects the fact that the thermal expansion coefficient is gradually reduced with the increase of reference pressure.

For the whole depth range in the ocean, a Matlab program sw_spicity.m is defined, which uses the in-situ pressure to linearly interpolate between spicity subroutines defined above for different reference pressures. One can also use the individual Matlab program sw_spcity_N.m to examine the spicity distribution over certain pressure range near the reference pressure as denoted by $\mathrm{N}$ (in unit of $1000 \mathrm{db}$ ).

The isopleths of potential density and potential spicity on several reference pressure levels are shown in Figure 1. It is clear that these two families of isopleths are nearly perpendicular to each other.

Although the zero point of spicity is defined for the water parcel with the mean temperature and salinity at each pressure level, the area-mean spicity on each pressure level is not zero, as shown in Figure 2. It is readily seen that in the upper ocean and at each pressure level the mean spiciness is strongly biased to the positive value according to both the definition by Flament (2002) and the new definition introduced in this study, i.e. there are more spicy water parcels than nonspicity water parcels. However, for the spicity defined in this study, the mean spicity at each pressure level in the upper ocean is close to zero. This fact suggests that spicity defined in this study can give a better description of how spicy the water parcels are on each pressure level.

Note that there is still a noticeable nonzero mean spicity on each pressure level. This fact of the nonzero mean spicity is due to the nonlinearity of the equation of state. In particular, thermal expansion coefficient $\alpha$ is very small for seawater near the freezing point, but it increases with temperature. As a result, the spacing between spicity-isolines is large for cold and fresh water, but it declines when both temperature and salinity increase, as shown in Figure 1. Assume that the mean temperature and salinity at a given pressure level $p_{0}$ are 
Horizontal mean spicity/spiciness

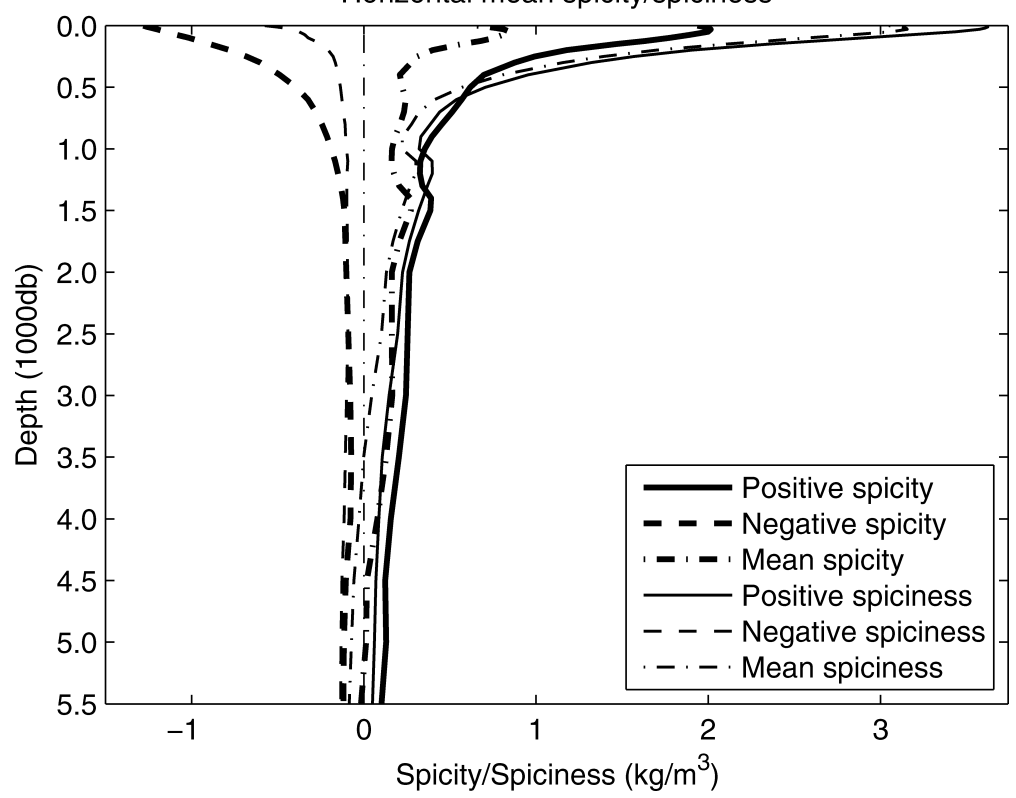

Figure 2. Vertical profile of the area-mean spicity and spiciness averaged on each pressure level, based on WOA09 data.

$\left(T_{0}, S_{0}\right)$, by definition, the corresponding spicity $\pi_{p_{0}}\left(S_{0}, T_{0}, p_{0}\right)=0$ is zero. There are two water parcels $P_{-}$and $P_{+}$, with corresponding temperature and salinity $\left(T_{0}-\Delta T, S_{0}-\Delta S\right)$ and $\left(T_{0}+\Delta T, S_{0}+\Delta S\right)$. Due to the nonlinearity of the equation of state, it is readily seen that

$$
\pi_{p_{0}}\left(S_{0}+\Delta S, T_{0}+\Delta T, p_{0}\right)>\left|\pi_{p_{0}}\left(S_{0}-\Delta S, T_{0}-\Delta T, p_{0}\right)\right|
$$

or

$$
\pi_{p_{0}}\left(S_{0}+\Delta S, T_{0}+\Delta T, p_{0}\right)+\pi_{p_{0}}\left(S_{0}-\Delta S, T_{0}-\Delta T, p_{0}\right)>0 .
$$

Therefore, using the new definition introduced in this study still gives rise to a positive-bias mean spicity on each pressure level.

\section{Application of spicity to the world oceans}

This newly defined spicity can be used to plot seawater properties on sections and quasihorizontal maps, as will be shown shortly. First, we start with a meridional section, based on data taken from WOA09 climatology (see Fig. 3).

In Figure 3a, heavy solid contours indicate spicy water (warm and salty water) and light solid contours indicate nonspicy water (cold and fresh water). Here the warm or cold is 
a) Spicity along $30.5^{\circ} \mathrm{W}$

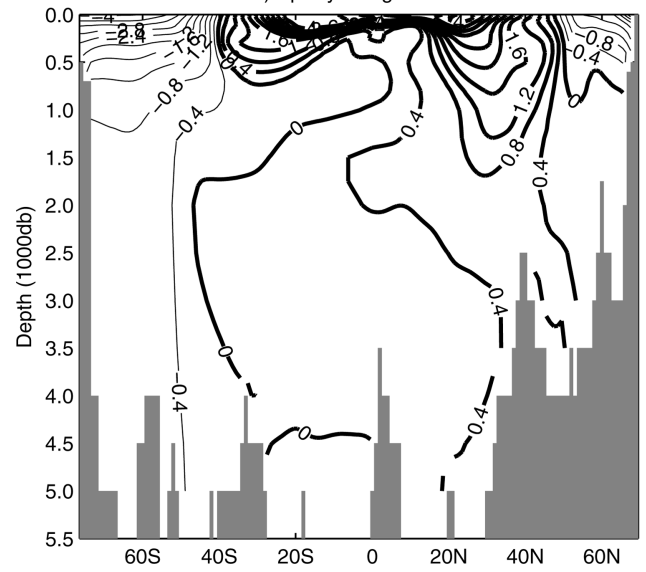

b) Spiciness along $30.5^{\circ} \mathrm{W}$

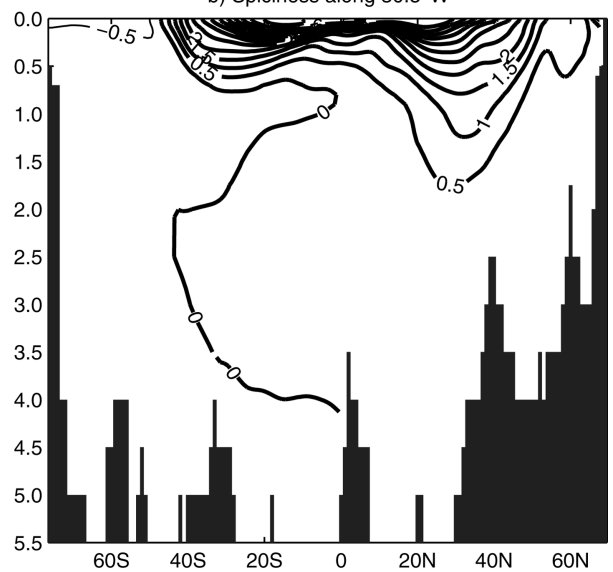

Figure 3. A meridional section along $30.5^{\circ} \mathrm{W}$, including both the distribution of spicity defined in this study (panel a) and spiciness (panel b) defined by Flament (2002).

defined as the deviation from the global mean temperature at the corresponding levels. Thus, this newly defined spicity provides a very clear indication of the spicity as referred to each pressure level. The cold and relatively fresh water at high latitudes can be seen clearly in this figure. In particular, the special water mass properties associated with the Antarctic Circumpolar Current are outstanding in the spicity contour plot, where a subsurface spicity minimum associated with the Antarctic Intermediate Water can be identified around the depth range of $500-1500 \mathrm{db}$ in the Southern Hemisphere. Such a clear picture is rather difficult to identify from the old definition postulated by Flament (2002).

Over the depth range of $1500-4500 \mathrm{db}$ the Atlantic Ocean is mostly controlled by salty and relatively warm water from the North Atlantic Ocean. However, the abyssal part of the South Atlantic Ocean is primarily controlled by the relatively cold and fresh water from the Antarctic seen as the low spicity water in this figure. Note that for the abyssal ocean both the old and new definition produce similar features because the old definition is based on using zero degree as the reference temperature, and for the abyssal ocean this is not too far away from the local mean value.

The application of this definition of spicity to a zonal section in the Southern Hemisphere, (Fig. 4), also provides a picture which reveals more information, compared with the spiciness defined by Flament (2002). The map of spicity reveals tongue-like subsurface features in all three basins. These features should be interpreted as the poleward transport of warm, salty upper waters from the subtropical gyres along the western boundary currents.

In addition, in the eastern Pacific there is the unique feature of a negative spicity columnar signal going all the way down to intermediate depths. This can be linked to the Subantarctic Mode Water (SAMW) in this area. The low value of spicity is a clear sign for the relatively low temperature induced by the well-known winter surface cooling, which leads to 

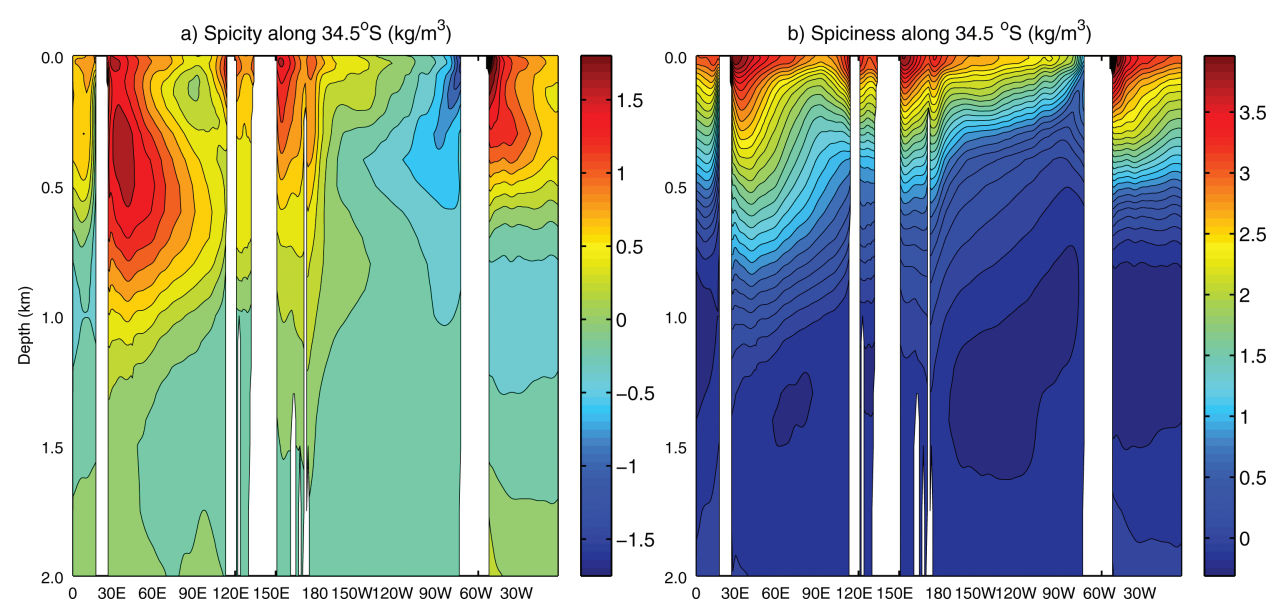

Figure 4. Spicity (a) and spiciness (b) distributions along the $34.5^{\circ} \mathrm{S}$ section in the Southern Hemisphere.

subsurface convection, a major mechanism responsible for the formation of SAMW. In fact, there is low spicity water at the middle depth (approximately at $1500 \mathrm{~m}$ ) in the eastern part of all three major basins, which indicates the signal of the Antarctic Intermediate Water (AAIW). These low spicity features suggest the northward advection of the low-salinity water, which is slightly colder than the main thermocline.

These features, however, are not clearly shown in the spiciness map. In particular, the subsurface spicity maximum in the South Indian Ocean sector cannot be seen in the spiciness section. In fact, the spiciness declines with depth, and there is no subsurface maximum or minimum. This figure clearly shows the advantage of using the newly defined spicity, which is based on the local pressure level.

As another example, the distribution of spicity along a meridional section along $179.5 \mathrm{~W}$ based on WOA09 data is shown in Figure 5. Typically, spicity has high positive values at low latitudes and high negative value at high latitudes. In fact, this spicity section map clearly shows the contrast between the warm and salty water at low and middle latitudes and the cold and fresh water at high latitudes. It is clearly seen that spicity is the thermodynamic variable which can indicate the most outstanding difference of water masses in the meridional section.

Spicity has been used as a very important index for the study of double diffusion in many previous studies. Therefore, spicity is closely linked to the different roles of temperature and salinity in the thermohaline circulation, and such roles are directly connected to the different diffusivity of temperature and salinity for different length scales of motion.

In order to explore the physics of quasi-horizontal thermohaline perturbations we can plot the water mass property distributions on isopycnal surfaces. There are many possible choices for the isopycnal surface, including the potential density surface and the so-called neutral surface. Due to the nonlinear equation of state for seawater, no exact neutral surface can be defined for the World Oceans. As a result, there are only approximate neutral surfaces. 


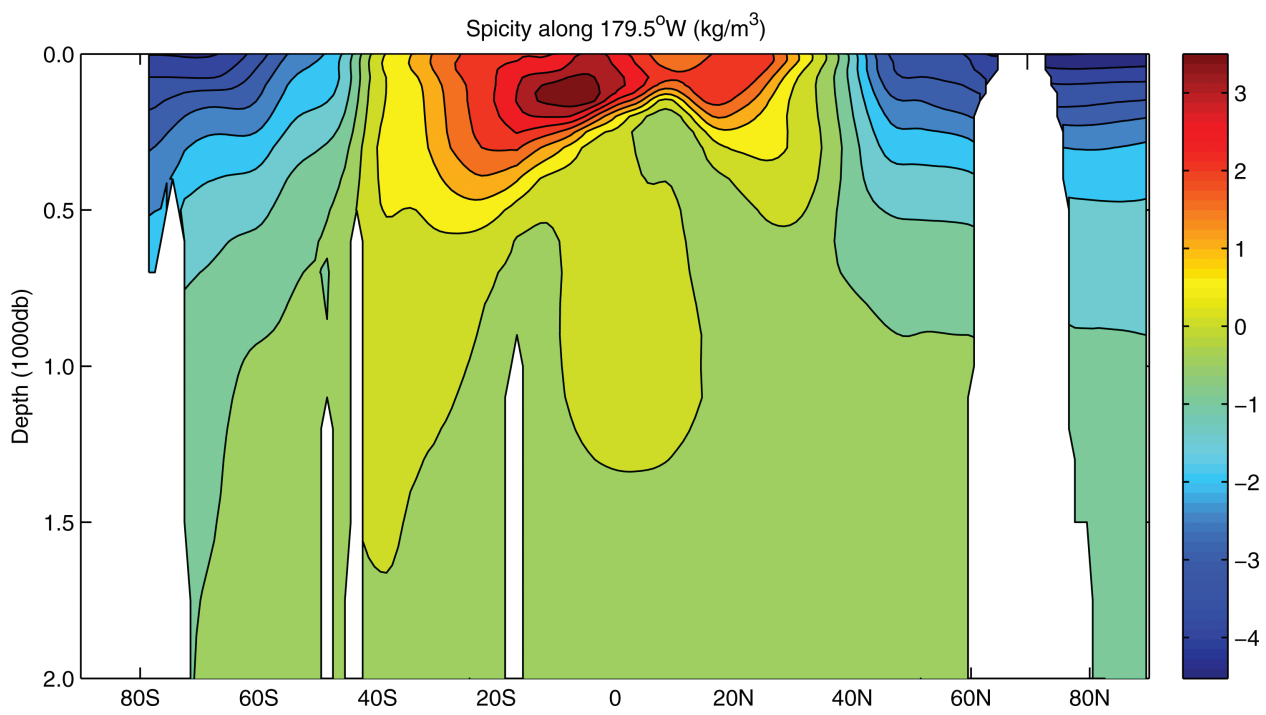

Figure 5. Spicity distribution along the $179.5^{\circ} \mathrm{W}$ section, based on WOA09 data.

The other choice is to use the potential density surface. For the upper ocean, over the depth range of $0-1000 \mathrm{db}$, the best choice is the potential density $\sigma_{0.5}$, which is defined using $500 \mathrm{db}$ as the reference pressure. The additional advantage of using a potential density surface is that potential density is a conserved quantity during idealized isentropic motions.

This potential density surface intersects the main thermocline in the subtropical basins of both the North Pacific Ocean and North Atlantic Ocean at the depth range of 500-800 db, as shown in Figure 6. In the Southern Hemisphere, it is slightly deeper, in particular in the Indian Ocean sector. This potential density surface also intersects the subpolar basins and the strong fronts associated with the Antarctic Circumpolar Current. Note that due to the strong zonal currents in the Southern Oceans, this isopycnal surface outcrop along the latitude of $50^{\circ} \mathrm{S}$. However, in the Northern Hemisphere there is no such strong zonal currents; thus, this potential density surface extends all the way to the Arctic Ocean without outcropping.

Since this is a constant potential density surface, thermohaline processes taking place on this surface must depend on something other than the density. Thus, the spicity may provide useful information for these thermohaline processes. The distribution of both potential temperature and salinity on this surface provides remarkable information: there are strong property gradients in the North Atlantic and North Pacific basins. The combination of these two basins indicates a strong contrast of thermohaline property difference between the North Pacific Ocean and North Atlantic Ocean.

There is a great contrast of thermohaline circulation between the North Pacific and North Atlantic oceans. It is well-known that there is strong meridional overturning circulation and deep water formation in the Atlantic Ocean. On the other hand, there is no deep water 
Depth $(\mathrm{db})$ of $\sigma_{0.5}=29.5\left(\mathrm{~kg} / \mathrm{m}^{3}\right)$

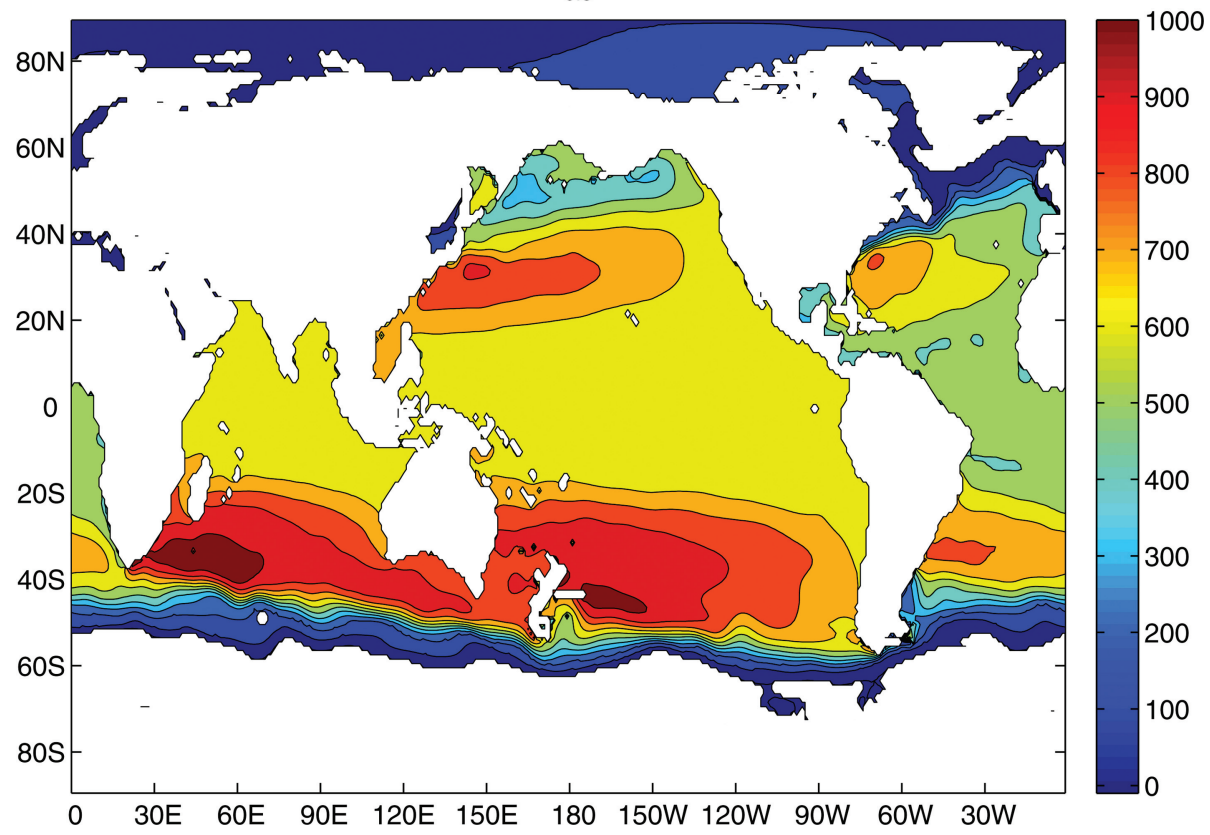

Figure 6. Depth (in units of $\mathrm{db}$ ) of potential density surface $\sigma_{0.5}=29.5 \mathrm{~kg} / \mathrm{m}^{3}$, based on WOA09 data.

formation in the North Pacific Ocean, and the corresponding meridional overturning circulation there is very weak, if there is any. The difference in thermohaline circulation is reflected in water mass properties. The North Atlantic Ocean is characterized by warm and salty water, while the North Pacific Ocean is characterized by cold and fresh water. The contrast of temperature and salinity between these two basins is clearly shown in Figures 7 and 8 .

The great difference in potential temperature and salinity in these two basins gives rise to remarkable difference in spicity as shown in Figure 9. High value of spicity in the North Atlantic Ocean has been linked to strong salt finger activity there. In addition, there is another positive spicity region in the Arabic Sea, which is obviously linked to salty water outflow of the Red Sea Overflow Water. The high spicity in this region suggests another region of high salty finger activity. Negative spicity in the North Pacific means very little salt finger activity there and the possibility of diffusive layering. However, the meaning of large horizontal gradient of spicity on this quasi-horizontal surface has not been explored, and thus it remains unclear.

\section{Conclusion}

We re-examine the concept of spiciness and introduce a new term, spicity. A new set of Matlab codes is developed which is subjected to the two principles. First, the isopleths 
Potential temperature $\left({ }^{\circ} \mathrm{C}\right)$ on $\sigma_{0.5}=29.5\left(\mathrm{~kg} / \mathrm{m}^{3}\right)$

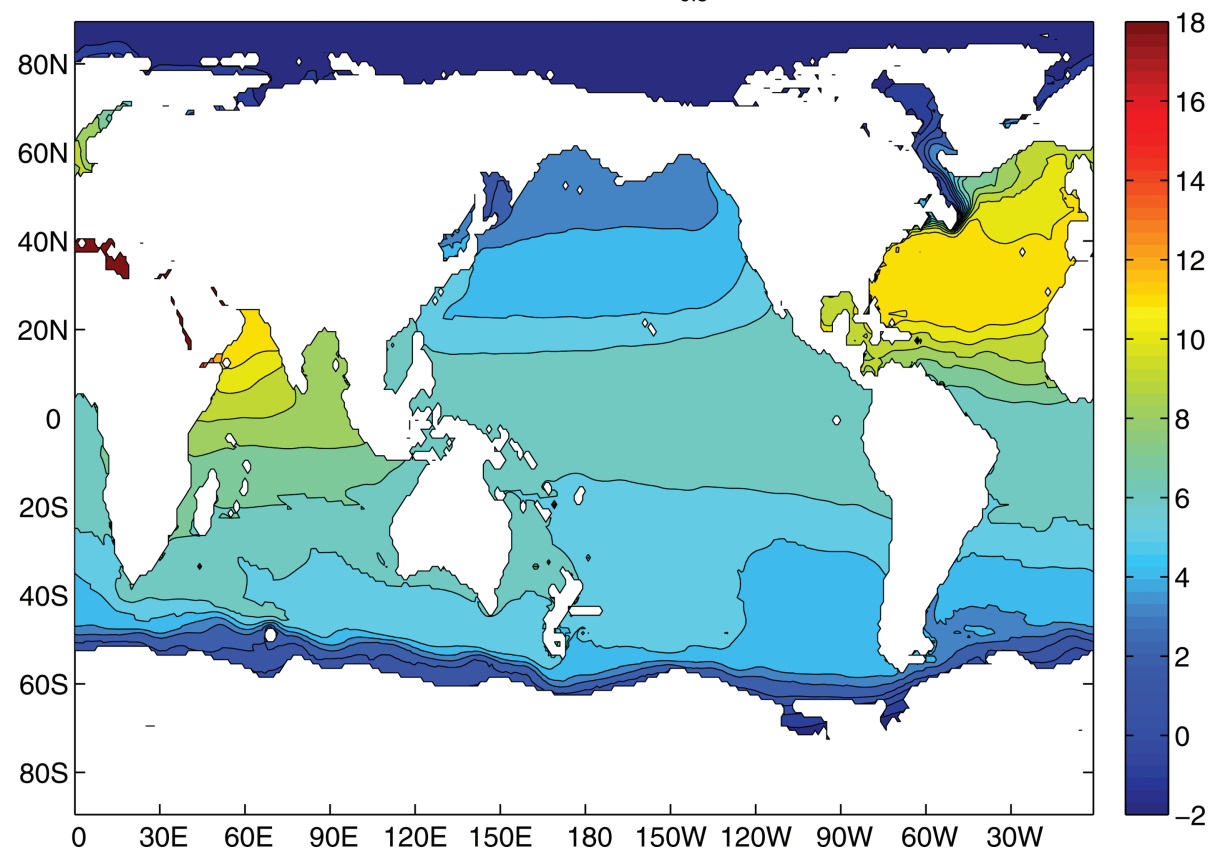

Figure 7. Potential temperature distribution on potential density surface $\sigma_{0.5}=29.5 \mathrm{~kg} / \mathrm{m}^{3}$, based on WOA09 data.

Salinity on $\sigma_{0.5}=29.5\left(\mathrm{~kg} / \mathrm{m}^{3}\right)$

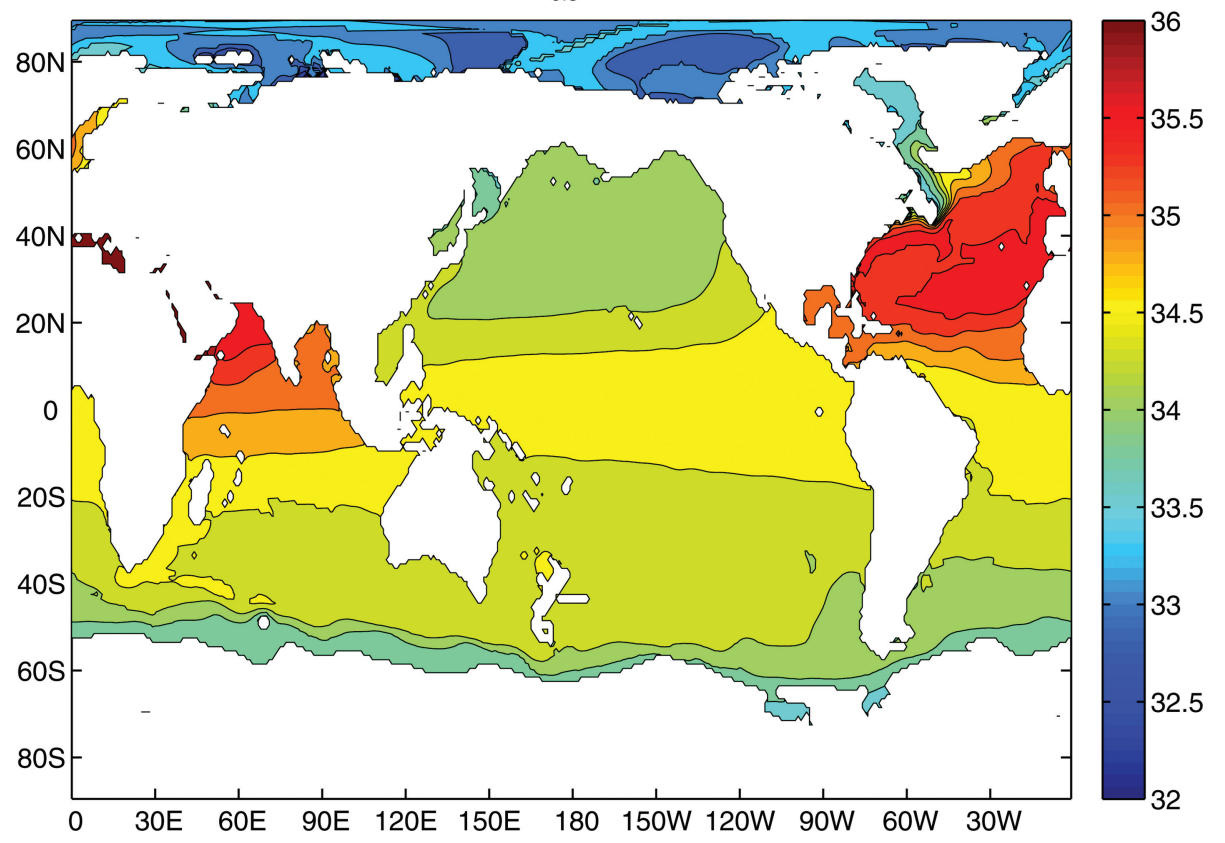

Figure 8. Salinity distribution on potential density surface $\sigma_{0.5}=29.3 \mathrm{~kg} / \mathrm{m}^{3}$, based on WOA09 
Spicity on $\sigma_{0.5}=29.5\left(\mathrm{~kg} / \mathrm{m}^{3}\right)$

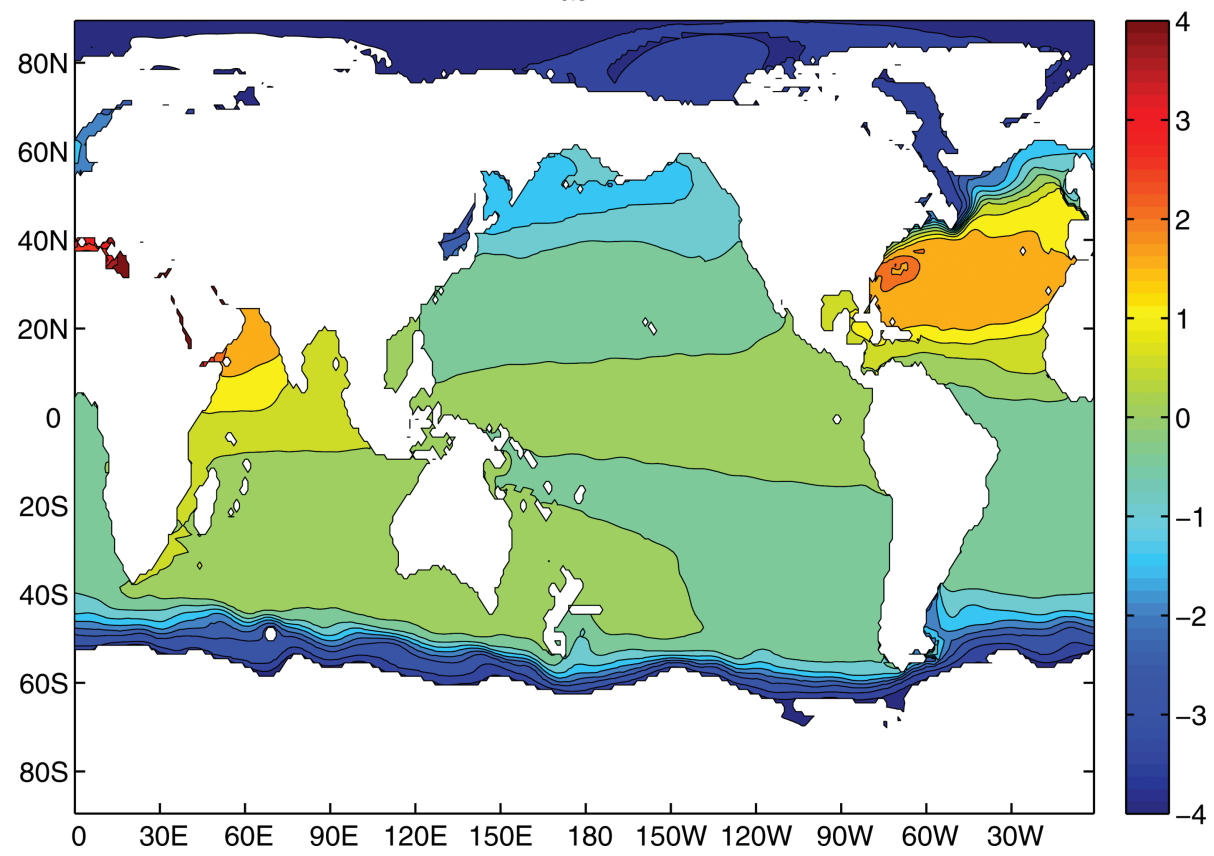

Figure 9. Spicity (in units of $\mathrm{kg} / \mathrm{m}^{3}$ ) distribution on potential density surface $\sigma_{0.5}=29.5 \mathrm{~kg} / \mathrm{m}^{3}$, based on WOA09 data.

of spicity should be orthogonal to those of density. Second, the zero point of spicity is defined more carefully. The ocean is separated into many pressure levels vertically. At each pressure level the zero point of spicity is set to the water mass with the mean salinity and temperature at the given reference pressure. This new definition of spicity gives more accurate information regarding whether a water mass can be considered as warm/salt or cold/fresh compared to other water masses on the same pressure level; thus, it is a better tool in describing the water mass properties associated with the thermohaline circulation.

Acknowledgments. I am grateful to Drs. Wei Wang and Ray Schmitt for their suggestions and stimulating discussions. The insightful comments from the editor and reviewers helped greatly to improve the presentation of the relevant issues. For many years the Geophysical Fluid Dynamics Program at WHOI has been an integral part of summer in Woods Hole. Therefore, this paper is dedicated to Melvin for his profound contribution to the program and his inspiring lectures.

\section{REFERENCES}

Antonov, J. I., D. Seidov, T. P. Boyer, R. A. Locarnini, A. V. Mishonov, H. E. Garcia, O. K. Baranova, M. M. Zweng, and D. R. Johnson. 2010. World Ocean Atlas 2009, Volume 2: Salinity, S. Levitus, ed., NOAA Atlas NESDIS 69, U.S. Government Printing Office, Washington, DC, 184 pp. 
Flament, P. 2002. A state variable for characterizing water masses and their diffusive stability: spiciness. Progr. Oceanogr., 54, 493-501.

Jackett, D. R. and T. J. McDougall. 1985. An oceanographic variable for the characterization of intrusions and water masses. Deep-Sea Res., 32, 1195-1207.

Locarnini, R. A., A. V. Mishonov, J. I. Antonov, T. P. Boyer, H. E. Garcia, O. K. Baranova, M. M. Zweng, and D. R. Johnson. 2010. World Ocean Atlas 2009, Volume 1: Temperature, S. Levitus, ed, NOAA Atlas NESDIS 68, U.S. Government Printing Office, Washington, DC, 184 pp.

Munk, W. 1981. Internal waves and small-scale processes, in Evolution of Physical Oceanography, MIT Press, Cambridge, MA, 264-291.

Rudnick, D. L. and J. P. Martin. 2002. On the horizontal density ratio in the upper ocean. Dyn. Atmos. Oceans, 36, 3-21.

Schmitt, R. W. 1994. Double diffusion in oceanography. Annu. Rev. Fluid Mech., 26, 255-85.

Stern, M. E. and J. S. Turner. 1969. Salt fingers and convecting layers. Deep-Sea Res., 16, 497-511.

Stommel, H. 1962. On the cause of the temperature-salinity curve in the ocean. Proceedings of the National Academy of Science USA, 48, 764-766.

Veronis, G. 1972. On properties of seawater defined by temperature, salinity and pressure. J. Mar. Res., 30, 227-255.

Received: 1 February, 2011; revised: 2 June, 2011. 